Masahiko Katayama

Akitoshi Ishizaka

Michiie Sakamoto

Seitaro Fujishima

Kiyotoshi Sekiguchi

Koichiro Asano

Tomoko Betsuyaku

Toru Kotani

Lorraine B. Ware

Michael A. Matthay

Satoru Hashimoto

\title{
Laminin $\gamma 2$ fragments are increased in the circulation of patients with early phase acute lung injury
}

L. B. Ware

Allergy, Pulmonary and Critical Care

Medicine, Department of Medicine,

Vanderbilt University, Nashville, TN, USA

M. A. Matthay

Cardiovascular Research Institute,

University of California,

San Francisco, CA, USA

S. Hashimoto

Department of Intensive Care Medicine, Kyoto Prefectural University of Medicine, Kyoto, Japan

A. Ishizaka · K. Asano

Department of Pulmonary Medicine,

Keio University School of Medicine,

Shinjuku, Tokyo, Japan

M. Sakamoto

Department of Pathology,

Keio University School of Medicine,

Shinjuku, Tokyo, Japan

S. Fujishima

Department of Emergency and Critical Care

Medicine, Keio University School of

Medicine, Shinjuku, Tokyo, Japan

K. Sekiguchi

Institute for Protein Research,

Osaka University, Suita, Osaka, Japan

T. Betsuyaku

First Department of Medicine,

Hokkaido University School of Medicine,

Sapporo, Hokkaido, Japan

T. Kotani

Department of Anesthesiology,

Tokyo Women's Medical University,

Shinjuku, Tokyo, Japan

\begin{abstract}
Objective: Laminin-5, a cell adhesive molecule expressed solely by epithelium, is known to enhance epithelial cell migration and repair of injured epithelium, after its essential component $\gamma 2$-chain is processed proteolytically. Our previous study revealed circulating levels of amino-terminal fragment of laminin $\gamma 2$-chain $(\mathrm{G} 2 \mathrm{~F})$ reflect epithelial tumor invasiveness in carcinoma patients, but its physiological role in alveolar epithelial injury remains unknown. Design: Sampling of epithelial lining fluids or pulmonary edema fluids from patients with acute lung injury (ALI) or related diseases was performed. Plasma samples were obtained from them at the time of disease onset or later. G2F concentrations were determined by immunoassay constructed by
\end{abstract}

ourselves. Results: We found a significantly higher amount of G2F in pulmonary edema and epithelial lining fluids of patients with ALI, as compared with those with the other respiratory diseases. Their plasma levels were also elevated significantly early at the onset of ALI (mean $\pm \mathrm{SD} ; 147 \pm 82 \mathrm{ng} / \mathrm{ml}$ in non-surviving and $90 \pm 56$ in surviving patients) as compared with those in the patients with cardiogenic pulmonary edema $(59 \pm 36)$ or idiopathic pulmonary fibrosis $(37 \pm 17)$, indicating alveolar epithelium rapidly secrete laminin-5 in ALI. At 5 days after onset, non-surviving patients maintained higher plasma concentrations $(152 \pm 84)$, but in contrast, the levels in surviving patients declined (71 \pm 35 ), suggesting secretion of laminin-5 was suppressed, associated with recovery from ALI.

Conclusion: Circulating G2F may be a biomarker for alveolar laminin-5 secreted early at disease onset in ALI, potentially regulating alveolar re-epithelialization.

Keywords Laminin - Circulation · Acute lung injury $\cdot$ Alveolar epithelial repair 


\section{Introduction}

The alveolar epithelium provides a primary defensive barrier to the external environment, and thus is frequently injured by inflammatory or physical stimuli in acute respiratory distress syndrome (ARDS) or acute lung injury (ALI) [1]. Repair of damaged alveolar epithelia is an indispensable step in restoring the lung to its normal architecture [2,3]. The repair process is initiated by the proliferation, migration and spreading of alveolar epithelial cells from the intact margins into the damaged areas $[4,5]$. Proliferation and differentiation of alveolar type-II cells are also important mechanisms in the reconstitution of extracellular matrix (ECM) that can promote alveolar epithelial repair [6, 7].

Laminins (LMs) are a family of high molecular weight ECM proteins, deposited in the basement membranes, also involved in cellular adhesion, growth and differentiation $[8,9]$. There are at least 15 types of LM isoforms, resulting from a variety of combinations of $\alpha$, $\beta$ and $\gamma$ chains $[9,10]$. Laminin-5 (LM5), also recently designated as laminin-332 [10], is a heterotrimer composed of $\alpha 3, \beta 3$ and $\gamma 2$ chains. LM5 is secreted solely by epithelial cells and plays an important role in epithelial cell migration especially during tumor invasion, metastasis or remodeling of epithelial tissue [11-14]. LM5 enhances cellular migration when it is simultaneously secreted into the ECM and activated via cleavage of its essential constituent $\gamma 2$-chain by matrix metalloproteinases (MMPs) $[15,16]$. This proteolysis can release the amino-terminal fragment of $\gamma 2$-chain (G2F), which is not deposited in the ECM, and can be subsequently detected in the peripheral blood [17]. We have developed an immunoassay for quantification of $\mathrm{G} 2 \mathrm{~F}$ in biological fluids and demonstrated that G2F is present in normal circulation. G2F concentrations in circulation correlated with the degree of carcinoma invasion in vivo, suggesting the potential clinical value of this circulating fragment as a biomarker for epithelial invasion induced by LM5 [17].

LM5 is potentially involved in normal epithelial repair [9, 18], and also expressed during epidermal wound healing at the leading edge of the dermal-epidermal junction by keratinocytes within several hours after mechanical injury [19]. Previous studies have shown that migration of alveolar epithelial cells is necessary during the repair process of ALI, which requires several hours for initiation and several days to be completed [3,20]. However, little is known about the cell migration factors produced or activated during injury, proliferation and differentiation of alveolar epithelium [21]. Therefore, we examined the relationship between disease status and alveolar LM5 accumulation during the exudative phase in patients with ALI, as reflected by circulating G2F levels in several biological fluids.

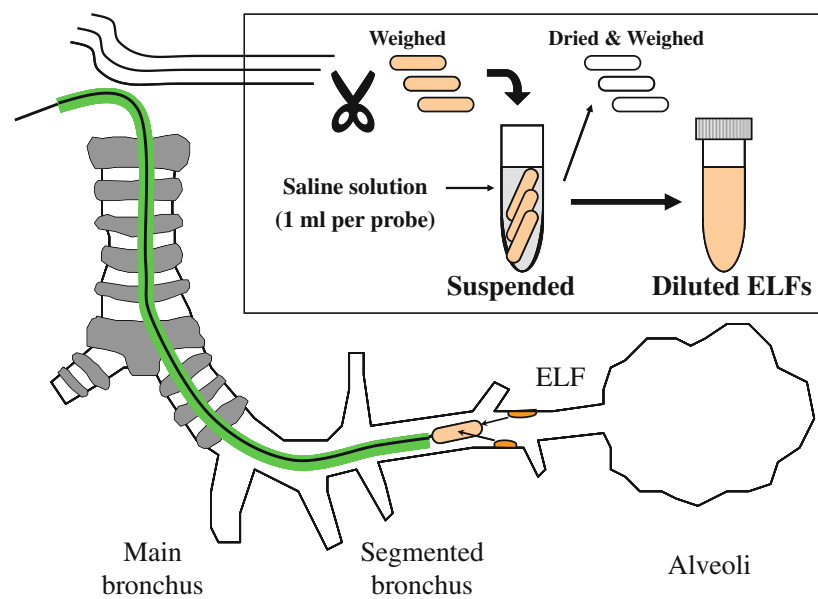

Fig. 1 Schematic representation of ELF collection procedure. Detailed procedure is described in "Materials and methods." Briefly, a flexible fiberoptic bronchoscope was inserted into the lungs. The bronchial micro-sampling probe, with the cotton probe as absorptive materials, was inserted into the lungs through the bronchoscope for absorbing the ELFs. The wet inner probe was sectioned, introduced in a pre-weighed tube and weighed. The diluted solution for measurements of biochemical constituents was prepared by adding $3 \mathrm{ml}$ of saline to the tube containing the triplicate probes. The probe was then dried and weighed again to measure the ELF volume recovered, and the dilution factor was calculated

\section{Materials and methods}

Collection of epithelial lining fluid (ELF)

and pulmonary edema fluid (PEF) specimens

ELF specimens were prepared according to the published procedure [22], as shown schematically in Fig. 1. The protocol was briefly described. Following routine premedication, a flexible fiberoptic bronchoscope (Olympus Co., Japan) was inserted into the lungs. The fiberscope was wedged in a segmental bronchus of the right middle lobe. The micro-sampling probe, with the cotton probe as absorptive material, was inserted into the lungs through the bronchoscope, and the inner probe was slowly advanced into the distal airway for absorbing the ELFs. The inner probe was removed, and the tip was placed in a pre-weighed tube, weighed and stored at $-80^{\circ} \mathrm{C}$. The diluted solution for G2F measurements was prepared by adding $3 \mathrm{ml}$ of saline to the tube containing the triplicate probes. The probe was then dried and weighed again to measure the ELF volume recovered, and the dilution factor was calculated.

Undiluted PEF samples were collected as previously described $[1,23]$. Briefly, the PEF sample was obtained within 15 min of intubation and mechanical ventilation. A 14-French suction catheter (Becton-Dickinson, NJ) was passed through the endotracheal tube and wedged into the distal airways; then, gentle suction was applied to obtain 
PEF (at least 1-2 ml), which was collected into a specimen trap, and 10-100 $\mathrm{U}$ of heparin was added to each sample. Then, the heparinized samples were centrifuged, and supernatants were stored at $-80^{\circ} \mathrm{C}$.

Subjects for analysis of G2F levels in ELFs and plasma specimens

Twenty-seven patients with ALI who were admitted between 1999 and 2001 to the Intensive Care Unit of Kyoto Prefectural University of Medicine Hospital or Keio University Hospital were enrolled into the first part of the study. ALI and ARDS were diagnosed using the American European Consensus Conference definitions [24]. Eligible patients met consensus conference oxygenation and radiographic criteria for either ALI or ARDS, and were followed until death or hospital discharge (survival). The lung injury score was calculated according to the published algorithms [25].

Plasma anticoagulated with EDTA was collected at onset (day 0) and at fixed intervals afterwards (days 1, 3, 5 and 7). ELF specimens were obtained only at day 0 as described above. Control plasma samples were collected from 15 healthy volunteers (11 males, 4 females), while control ELF samples were obtained from 13 males and 2 females who underwent bronchoscopy to identify causes of hemoptysis, or to examine small, solitary, peripheral pulmonary nodules. Normal and control samples were also obtained between 1999 and 2001 at the above hospitals.

Plasma and ELF specimens were also collected from 5 patients with cardiogenic pulmonary edema (CPE) and 11 patients with idiopathic pulmonary fibrosis (IPF) who were recruited from the Tokyo Women's Medical University Hospital or the Keio University Hospital, respectively. Plasma specimens were separately obtained from 20 sepsis patients without ALI, recruited from Iwate Medical University Hospital, diagnosed according to published guidelines [26]. Additionally, ELF specimens were also obtained from 12 patients with chronic obstructive pulmonary disease (COPD), recruited from the Hokkaido University of Medicine Hospital. All of these samples were collected between 1999 and 2006. The institutional review board of each center in Japan approved the study protocol, and written informed consent was obtained from either the patient or each patient's next of kin or legal representative before enrollment.

\section{Subjects for analysis of G2F levels in PEF specimens}

In the second part of the study, 21 patients with ALI/ ARDS as defined above and 11 control patients with CPE were included in this study [24, 27]. In order to allow comparison of mean concentrations of G2F in ELFs and
PEFs, samples of undiluted PEF were obtained only at the onset of respiratory failure. These patients were enrolled at the Moffitt Long University and San Francisco General Hospitals in San Francisco between 1990 and 2005. The institutional review board at University of California at San Francisco approved the study protocol, and written informed consent was obtained from either the patient or each patient's next of kin or legal representative before enrollment. The lung injury score was also calculated [25].

\section{Immunoassay}

The obtained specimens were stored at $-80^{\circ} \mathrm{C}$ until usage. The concentration of soluble G2Fs was determined as described previously [17].

Statistical analysis

Between-group statistical comparisons were performed on mean $\mathrm{G} 2 \mathrm{~F}$ concentration data using one-way analysis of variance (ANOVA), followed by a Bonferroni/Dunn post-hoc test. Time course data of plasma G2F levels were also analyzed using repeated measures ANOVA. Statistical differences for mean PEF concentrations of G2F between CPE and ALI patient groups were analyzed using non-parametric statistics (Mann-Whitney $U$ test). Differences were regarded as statistically significant when $P<0.05$. All calculations were performed using StatView 5.0 software (SAS Institute Inc., Cary, NC).

\section{Results}

G2F levels in ELF samples of the ALI patients

In the first part of the study, we enrolled 27 patients with ALI. Nineteen patients survived to yield a hospital mortality rate of $30 \%$. Baseline clinical features of the surviving or non-surviving patients are shown in Table 1. ELF samples were collected from the 25 ALI patients (including 19 survivors and 6 non-survivors) at onset (day 0 ). One-way ANOVA revealed a significant difference in the ELF levels of G2Fs among patient and control groups $(F=6.3, P<0.0004)$. G2F levels in ELF from these patients were ten-fold higher (mean $\pm \mathrm{SD}$; $6,034 \pm 6,245 \mathrm{ng} / \mathrm{ml}$ ) than those from 15 control subjects $(813 \pm 807 \mathrm{ng} / \mathrm{ml}, \quad P<0.0003)$ or compared to 12 COPD patients $(350 \pm 442 \mathrm{ng} / \mathrm{ml}, P<0.0003)$, indicating a high degree of secretion of G2F into the air spaces in the early phase of ALI (Fig. 2). The ELF levels in these 25 ALI patients were also significantly elevated compared to 5 control patients with CPE $(1,237 \pm 807 \mathrm{ng} / \mathrm{ml}$, 

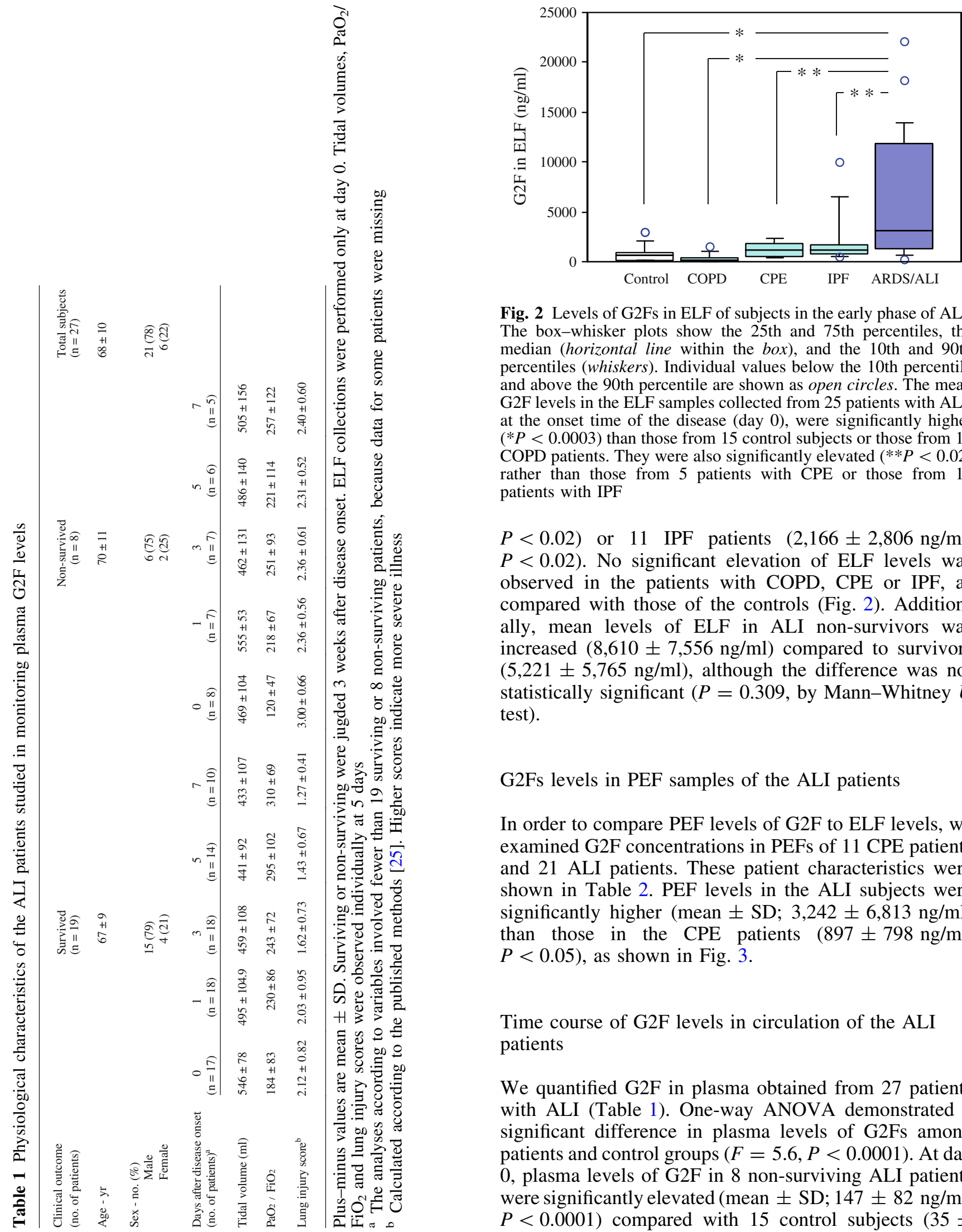

Fig. 2 Levels of G2Fs in ELF of subjects in the early phase of ALI. The box-whisker plots show the 25th and 75th percentiles, the median (horizontal line within the box), and the 10th and 90th percentiles (whiskers). Individual values below the 10th percentile and above the 90th percentile are shown as open circles. The mean G2F levels in the ELF samples collected from 25 patients with ALI, at the onset time of the disease (day 0), were significantly higher $(* P<0.0003)$ than those from 15 control subjects or those from 12 COPD patients. They were also significantly elevated $(* * P<0.02)$ rather than those from 5 patients with $\mathrm{CPE}$ or those from 11 patients with IPF

$P<0.02)$ or 11 IPF patients $(2,166 \pm 2,806 \mathrm{ng} / \mathrm{ml}$, $P<0.02$ ). No significant elevation of ELF levels was observed in the patients with COPD, CPE or IPF, as compared with those of the controls (Fig. 2). Additionally, mean levels of ELF in ALI non-survivors was increased $(8,610 \pm 7,556 \mathrm{ng} / \mathrm{ml})$ compared to survivors $(5,221 \pm 5,765 \mathrm{ng} / \mathrm{ml})$, although the difference was not statistically significant $(P=0.309$, by Mann-Whitney $U$ test).

\section{G2Fs levels in PEF samples of the ALI patients}

In order to compare PEF levels of G2F to ELF levels, we examined G2F concentrations in PEFs of 11 CPE patients and 21 ALI patients. These patient characteristics were shown in Table 2. PEF levels in the ALI subjects were significantly higher (mean $\pm \mathrm{SD} ; 3,242 \pm 6,813 \mathrm{ng} / \mathrm{ml}$ ) than those in the CPE patients $(897 \pm 798 \mathrm{ng} / \mathrm{ml}$, $P<0.05$ ), as shown in Fig. 3 .

Time course of G2F levels in circulation of the ALI patients

We quantified G2F in plasma obtained from 27 patients with ALI (Table 1). One-way ANOVA demonstrated a significant difference in plasma levels of G2Fs among patients and control groups $(F=5.6, P<0.0001)$. At day 0 , plasma levels of G2F in 8 non-surviving ALI patients were significantly elevated (mean $\pm \mathrm{SD} ; 147 \pm 82 \mathrm{ng} / \mathrm{ml}$, $P<0.0001)$ compared with 15 control subjects $(35 \pm$ 
Table 2 Comparison of physiological characteristics of the patient groups entered in the PEF study

\begin{tabular}{lll}
\hline Disease (no. of patients) & CPE $(n=11)$ & ALI $(n=21)$ \\
\hline Age (years) & $45 \pm 20$ & $42 \pm 14$ \\
Sex, no. (\%) & & \\
Male & $6(54)$ & $12(59)$ \\
Female & $5(46)$ & $9(41)$ \\
Caucasian, no. (\%) & $6(55)$ & $11(50)$ \\
Current smoker, no. (\%) & $1(9)$ & $7(32)$ \\
Sepsis, no. (\%) & $0(0)$ & $11(50)$ \\
Days of unassisted ventilation & $19 \pm 9$ & $7 \pm 10$ \\
Tidal volume (ml) & $677 \pm 166$ & $620 \pm 220$ \\
PaO $_{2} /$ FiO $_{2}$ & $143 \pm 90$ & $74 \pm 27$ \\
Lung injury score $^{\mathrm{a}}$ & $2.50 \pm 0.70$ & $3.10 \pm 0.50$ \\
$\mathrm{Hospital} \mathrm{mortality,} \mathrm{no.}(\%)$ & $2(18)$ & $15(68)$ \\
\hline
\end{tabular}

Plus-minus values are mean $\pm \mathrm{SD}$

Tidal volumes, $\mathrm{PaO}_{2} / \mathrm{FiO}_{2}$ and lung injury scores were observed the onset of the diseases

a Calculated according to the published methods [25]. Higher scores indicate more severe illness

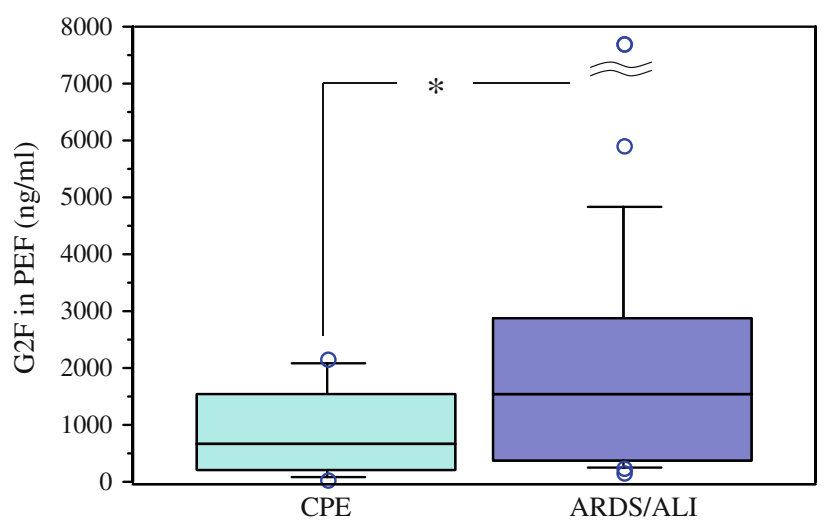

Fig. 3 Comparison of G2F levels in PEF of the patients with CPE and ALI. The box-whisker plots show the 25th and 75th percentiles, the median (horizontal line within the box), and the 10th and 90th percentiles (whiskers). Individual values below the 10th percentile and above the 90th percentile are shown as open circles. The mean G2F levels in the PEF samples collected from 21 patients with ALI were moderately higher $(* P<0.05)$ than those from $11 \mathrm{CPE}$ patients. The highest G2F value $(32,197 \mathrm{ng} / \mathrm{ml})$ was out of the range

$14 \mathrm{ng} / \mathrm{ml}$ ), as shown in Fig. 4. The plasma level of G2F in 17 surviving ALI patients at day 0 was moderately elevated $(90 \pm 56 \mathrm{ng} / \mathrm{ml})$ and was also significantly higher $(P<0.006)$ than control values. G2F levels were higher in plasma from ALI non-survivors than survivors at day 0 $(P<0.02)$, a difference that persisted on day $5(71 \pm$ $35 \mathrm{ng} / \mathrm{ml}$ for survivors vs. $152 \pm 84 \mathrm{ng} / \mathrm{ml}$ in non-survivors, $P<0.003)$ and day $7(57 \pm 26 \mathrm{ng} / \mathrm{ml}$ for survivors vs. $174 \pm 107 \mathrm{ng} / \mathrm{ml}$ for non-survivors, $P<0.0002$ ) (Fig. 4). Plasma G2F concentrations for each of the nonsurviving ALI patient groups at day $0,1,3,5$ or 7 were significantly higher than those for the control group

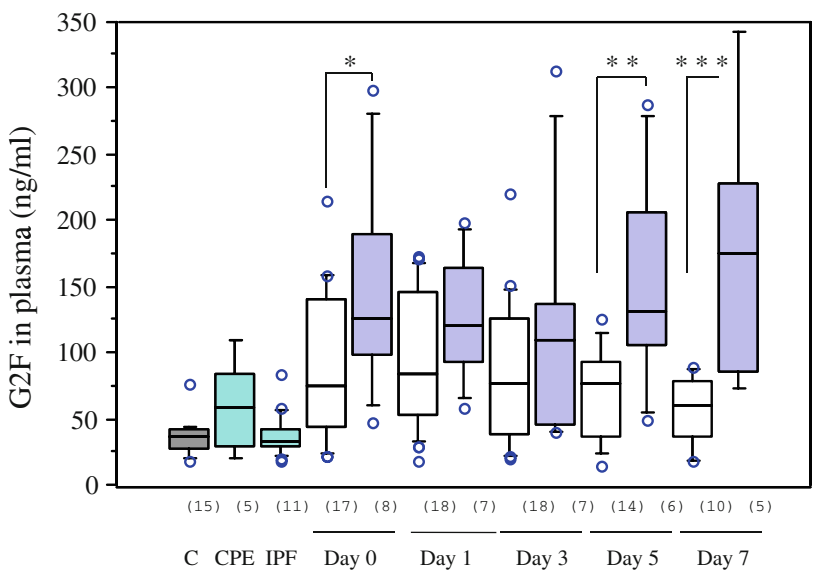

Fig. 4 Levels of G2Fs in plasma of clinical subjects. Number of the subjects in each column was indicated in parentheses. The boxwhisker plots show the 25th and 75th percentiles, the median (horizontal line within the box), and the 10th and 90th percentiles (whiskers). Individual values below the 10th percentile and above the 90th percentile are shown as open circles. The ALI patients were classified into the five groups (day $0,1,3,5$ or 7 after disease onset), and each of the groups was further divided into survivors (white box) and non-survivors (shadowed box). The plasma G2F levels of 15 control subjects were indicated as a black box in the left end. Statistical differences between survivors and non-survivors are indicated as follows: $* P<0.02, * * P<0.003$, or $* * * P<0.0002$. The levels in the patients with CPE or IPF were not significantly higher than those in the control subjects

$(P<0.0001$ on day 0,5 or $7 ; P<0.0004$ for day 1 ; $P<0.002$ for day 3 ). In contrast, plasma G2F concentrations in the surviving patient groups at day 0,1 or 3 were moderately elevated $(P<0.006$ for day $0 ; P<0.002$ for day $1 ; P<0.007$ for day 3 vs. control group), but no difference could be detected at day 5 or $7(P=0.08$ or 0.33 vs. control group, respectively). Plasma levels of G2Fs were not significantly elevated in the patients with CPE $(59 \pm 36 \mathrm{ng} / \mathrm{ml})$ or IPF $(37 \pm 17 \mathrm{ng} / \mathrm{ml})$, as compared with those in control subjects (Fig. 4).

By using repeated measures ANOVA, we also found that time course data of plasma G2F levels in non-survivors were statistically different from those in survivors $(P<0.02)$. Plasma G2F concentrations of the 20 patients with non-ALI sepsis were not high $(32 \pm 18 \mathrm{ng} / \mathrm{ml}$, not shown in Fig. 4), similar to levels in the controls $(35 \pm 14 \mathrm{ng} / \mathrm{ml})$.

\section{Discussion}

It is well understood that the degree of alveolar epithelial injury is an important predictor of the outcome in ARDS/ ALI [20, 28]. However, there are currently no reliable clinical or biological markers of the extent of alveolar epithelial injury. Therefore, we tested the pathogenetic significance of a novel biological molecule expressed in specific response to the early onset of ALI. 
Recent experimental data suggest that accelerating reepithelialization of the alveolar barrier leads to the resolution of ALI [3, 21, 28]. Re-epithelialization can occur even in the absence of the proliferation of epithelial cells or the recruitment of stem cells immediately after the injury $[3,4]$. Previous studies hypothesized that most of alveolar repair is mediated by type-II pneumocytes that migrate and differentiate into type-I cells under stimulation of some component of ECM proteins in newly deposited basement membranes [29]. We prospectively expected LM5 may be expressed simultaneously with type-II pneumocyte migration during the early phase alveolar epithelial repair after the onset of ALI.

The alveolar basement membrane contains several isoforms of LMs, and several functions for these adhesion molecules have been elucidated $[6,30]$. LM5 attracted our interest because it is specifically expressed in epithelium and stimulates cell migration, especially keratinocyte remodeling or carcinoma cell invasion [11-14]. Our prior experimental studies suggest that physiological epithelial cell migration can be estimated by the circulating levels of G2F, derived from the essential component $\gamma 2$-chains of LM5 [17]. Thus, we hypothesized circulating G2F levels are associated with alveolar epithelial migration to repair the injured lung.

Expression of LM5 in type-II pneumocytes was reported previously, but its function is unknown [30]. In this study, for the first time, we report that significant increases of G2F are found in the circulation of ALI patients at the onset of acute respiratory failure (Figs. 2, 3, 4). Therefore, we speculate that the alveolar epithelial cells acting to repair the injured sites should prominently express migration-active LM5, generated by proteolytic shedding of the G2F domain, and that soluble $\mathrm{G} 2 \mathrm{~F}$ is consequently released from the alveolar surface into the ELF and diffuses from the ECM into the circulation of ALI patients. The degree of G2F secretion at the onset of ALI may be independent of disease severity, as migration of alveolar epithelial cells may reflect the drive to repair damage per se, rather than the actual extent of injury. As satisfactory repair of the injured epithelium is achieved, recovery of function in patients responding to therapy is associated with a decrease of circulating G2Fs at day 5 and 7 (Fig. 4). In contrast, the plasma G2F levels in ALI patients unresponsive to therapy remained significantly elevated at days 5 or 7 , suggesting that some physiological signaling might operate to promote continued LM5 synthesis and proteolytic cleavage of its $\gamma 2$ chain by type-II pneumocytes in the absence of adequate tissue repair, since alveolar epithelial function was not sufficiently normalized in these cases (Fig. 4). We also found that mean G2F concentrations in ELF samples from the ALI patients are comparable with those in PEF specimens from another group of the ALI patients, supporting the conclusion that $\mathrm{G} 2 \mathrm{~F}$ levels in ELFs obtained during routine bronchoscopic monitoring can accurately reflect the quantity of them released into alveolar epithelial surfaces.
Approximately half of the ALI patients usually received pulse therapy of steroids, which may not affect their G2F levels (data not shown), because none of increased G2F was found in the patients with COPD or IPF probably treated with any steroids.

We should not be regardless of potential bias in our analysis using multiple specimens from six different medical centers, because their diagnosis cannot be always standardized. Our study is the initial step to estimate significance of circulating G2F in respiratory disorders, using rarely obtainable specimens such as ELF or PEF. Increase of G2Fs in the ELF demonstrated they are mainly derived from injured alveolar tissues, as well as their increase in the PEF. Some further trials without potential institutional bias will be recommended, probably assisted by our present study.

Immunohistochemical observation of LM5 $\gamma 2$ chains in IPF lung tissues has been already reported, demonstrating that regenerating epithelial cells in IPF can actually synthesize $\gamma 2$ chains, but their expression levels in IPF are significantly reduced than those in cryptogenic organizing pneumonia [31]. This evidence suggested that alveolar LM5 accumulation can be induced preferably at the early phase of pulmonary injury rather than during the chronic phase of IPF, corresponding with our present results that G2F levels in ALI patients at the disease onset were significantly higher than those in IPF patients (Figs. 2, 4). We have also preliminarily detected LM5 expression in small population of pneumocytes in ALI by immunohistochemical technique (unpublished observation), predicting G2Fs are endogenously secreted from injured alveolar epithelium. Some further studies are needed to investigate tissue expression of LM5 in the patients with ALI, whose circulating G2F increased significantly.

Several proteases may be secreted from inflammatory leukocytes as they transmigrate into injured lung tissue. If LM5 is deposited in alveolar ECM, it may be degraded into small fragments in ALI. However, circulating G2F levels appear to be independent of such random proteolysis, since G2F is unlikely to be deposited in epithelial ECM [32]. Shedding of G2F from LM5 heterotrimers in vivo is critically mediated by membrane-type 1 MMP, and LM5 processed by the other MMPs cannot promote epithelial cell migration $[16,33]$. We infer that the physiological action of LM5 to initiate epithelial repair may be imparted specifically by membrane-type $1 \mathrm{MMP}$ cleavage and not random proteolysis.

In conclusion, we report that circulating G2F increases substantially at disease onset in ALI patients. In the patients recovering from ALI, G2F concentrations declined in parallel with their clinical improvement; in contrast, prolonged elevation of G2F was associated with poorer prognosis, potentially suggesting that circulating G2F may be a useful biomarker to monitor the pneumocyte re-epithelialization process during alveolar epithelial repair in ALI/ARDS patients. 
Acknowledgments We thank Robert M. Senior (Washington University School of Medicine at Barnes-Jewish Hospital) for his excellent comments and Nobuhiro Sato (Iwate Medical University, Japan) for supplying sepsis samples, and also the nurses and physicians, as well as our patients and their families, who supported this study. This work was supported, in part, by a grant-in-aid for Fundamental Scientific Research from the Education Ministry of Japan 16390457 to Satoru Hashimoto, 07670678 to Akitoshi Ishizaka, and by National Institutes of Health grants HL70521 and HL081332 to Lorraine B. Ware, and HL51856 to Michael A. Matthay.
Conflict of interest statement None of the authors has a financial relationship with a commercial entity that has an interest in the subject of this manuscript.

Open Access This article is distributed under the terms of the Creative Commons Attribution Noncommercial License which permits any noncommercial use, distribution, and reproduction in any medium, provided the original author(s) and source are credited.

\section{References}

1. Matthay MA, Wiener-Kronish JP (1990) Intact epithelial barrier function is critical for the resolution for alveolar edema in humans. Am Rev Respir Dis 142:1250-1257

2. Dos Santos CC (2008) Advances in mechanisms of repair and remodelling in acute lung injury. Intensive Care Med 34:619-630

3. Geiser T (2003) Mechanisms of alveolar epithelial repair in acute lung injury - a translational approach. Swiss Med Wkly 133:586-590

4. Adamson IYR, Bowden DH (1974) The type 2 cell as progenitor of alveolar epithelial regeneration. A cytodynamic study in mice after exposure to oxygen. Lab Invest 30:35-42

5. Zahm JM, Chevillard M, Puchelle E (1991) Wound repair of human surface respiratory epithelium. Am J Respir Cell Mol Biol 5:242-248

6. Dunsmore SE, Rannels DE (1996) Extracellular matrix biology in the lung. Am J Physiol Lung Cell Mol Physiol 270:L3-L27

7. Crouch E (1990) Pathobiology of pulmonary fibrosis. Am J Physiol Lung Cell Mol Physiol 259:L159-L184

8. Colognato H, Yurchenco PD (2000) Form and function: the laminin family of heterotrimers. Dev Dyn 218:213-234

9. Tzu J, Marinkovich MP (2008) Bridging structure with function: structural, regulatory, and developmental role of laminins. Int $\mathbf{J}$ Biochem Cell Biol 40:199-214

10. Aumailley M, Bruckner-Tuderman L, Carter WG, Deutzmann R, Edgar D, Ekblom P, Engel J, Engvall E, Hohenester E, Jones JCR, Kleinman HK, Marinkovich MP, Martin GR, Mayer U, Meneguzzi G, Miner JH, Miyazaki K, Patarroyo M, Paulsson M, Quaranta V, Sanes JR, Sasaki T, Sekiguchi K, Sorokin LM, Talts JF, Tryggvason K, Uitto J, Virtanen I, von der Mark K, Wewer UM, Yamada Y, Yurchenco PD (2005) A simplified laminin nomenclature. Matrix Biol $24: 326-332$
11. Rousselle P, Lunstrum GP, Keene DR, Burgeson RE (1991) Kalinin: an epithelium-specific basement membrane adhesion molecule that is a component of anchoring filaments. J Cell Biol 114:567-576

12. Carter WG, Ryan MC, Gahr PJ (1991) Epiligrin, a new cell adhesion ligand for integrin $\alpha 3 \beta 1$ in epithelial basement membranes. Cell 65:599-610

13. Miyazaki K, Kikkawa Y, Nakamura A, Yasumitsu H, Umeda M (1993) A large cell-adhesive scatter factor secreted by human gastric carcinoma cells. Proc Natl Acad Sci USA 90:11767-11771

14. Fukushima Y, Ohnishi T, Arita N, Hayakawa T, Sekiguchi K (1998) Integrin $\alpha 3 \beta 1$-mediated interaction with laminin-5 stimulates adhesion, migration and invasion of malignant glioma cells. Int J Cancer 76:63-72

15. Giannelli G, Falk-Marzillier J, Schiraldi O, Stetler-Stevenson WG, Quaranta V (1997) Induction of cell migration by matrix metalloprotease- 2 cleavage of laminin-5. Science 277:225-228

16. Koshikawa N, Giannelli G, Cirulli V, Miyazaki K, Quaranta V (2000) Role of cell surface metalloprotease MT1-MMP in epithelial cell migration over laminin-5. J Cell Biol 148:615-624

17. Katayama M, Sanzen N, Funakoshi A, Sekiguchi K (2003) Laminin $\gamma 2$-chain fragment in the circulation: a prognostic indicator of epithelial tumor invasion. Cancer Res 63:222-229

18. Nguyen BP, Ryan MC, Gil SG, Carter WG (2000) Deposition of laminin 5 in epidermal wounds regulates integrin signaling and adhesion. Curr Opin Cell Biol 12:554-562

19. Ryan MC, Tizard R, VanDevanter DR, Carter WG (1994) Cloning of the LamA3 gene encoding the $\alpha 3$ chain of the adhesive ligand epiligrin. Expression in wound repair. $\mathrm{J}$ Biol Chem 269:22779-22787

20. Berthiaume Y, Lesur O, Dagenais A (1999) Treatment of adult respiratory distress syndrome: plea for rescue therapy of the alveolar epithelium. Thorax 54:150-160
21. Matthay MA, Zimmerman GA, Esmon C, Bhattacharya J, Coller B, Doerschuk CM, Floros J, Gimbrone MA, Hoffman E, Hubmayr RD, Leppert M, Matalon S, Munford R, Parsons P, Slutsky AS, Tracey KJ, Ward P, Gail DB, Harabin AL (2003) Future research directions in acute lung injury. Am J Respir Crit Care Med 167:1027-1035

22. Ishizaka A, Watanabe M, Yamashita T, Ogawa Y, Koh H, Hasegawa N, Nakamura H, Asano K, Yamaguchi K, Kotani M, Kotani T, Morisaki H, Takeda J, Kobayashi K, Ogawa S (2001) New bronchoscopic microsample probe to measure the biochemical constituents in epithelial lining fluid of patients with acute respiratory distress syndrome. Crit Care Med 29:896-898

23. Ware LB, Matthay MA (2001) Alveolar fluid clearance is impaired in the majority of patients with acute lung injury and the acute respiratory distress syndrome. Am J Respir Crit Care Med 163:1376-1383

24. Bernard GR, Artigas A, Brigham KL, Carlet J, Falke K, Hudson L, Lamy M, Legall JR, Morris A, Spragg R, the Consensus Committee (1994) The American-European Consensus Conference on ARDS: definitions, mechanisms, relevant outcomes, and clinical trial coordination. Am J Respir Crit Care Med 149:818-824

25. Murray JF, Matthay MA, Luce JM, Flick MR (1988) An expanded definition of the adult respiratory distress syndrome. Am Rev Respir Dis 138:720-723

26. Bone RC (1996) The sepsis syndrome. Definition and general approach to management. Clin Chest Med 17:175181

27. Verghese GM, Ware LB, Matthay BA, Matthay MA (1999) Alveolar epithelial fluid transport and the resolution of clinically severe hydrostatic pulmonary edema. J Appl Physiol 87:1301-1312

28. Ware LB, Matthay MA (2000) The acute respiratory distress syndrome. $\mathrm{N}$ Engl J Med 342:1334-1349 
29. Lwebuga-Mukasa JS (1991) Matrixdriven pneumocyte differentiation. Am Rev Respir Dis 144:452-457

30. Pierce RA, Griffin GL, Mudd MS, Moxley MA, Longmore WJ, Sanes JR, Miner JH, Senior RM (1998)

Expression of laminin $\alpha 3, \alpha 4$, and $\alpha 5$ chains by alveolar epithelial cells and fibroblasts. Am J Respir Cell Mol Biol 19:237-244
31. Lappi-Blanco E, Kaarteenaho-Wiik R, Salo S, Sormunen R, Määttä M, AutioHarmainen H, Soini Y, Pääkkö P (2004) Laminin-5 $\gamma 2$ chain in cryptogenic organizing pneumonia and idiopathic pulmonary fibrosis. Am J Respir Crit Care Med 169:27-33

32. Katayama M, Sekiguchi K (2004) Laminin-5 in epithelial tumour invasion. J Mol Histol 35:277-286
33. Pirilä E, Sharabi A, Salo T, Quaranta V, Tu H, Heljasvaara R, Koshikawa N, Sorsa T, Maisi P (2003) Matrix metalloproteinases process the laminin5 /2-chain and regulate epithelial cell migration. Biochem Biophys Res Commun 303:1012-1017 